\title{
The Infrared Nuclear Emission of Seyfert Galaxies on Parsec Scales: Testing the Clumpy Torus Models
}

\author{
C. Ramos Almeida ${ }^{1}$, N. A. Levenson ${ }^{2}$, J. M. Rodríguez Espinosa ${ }^{1}$, \\ A. Alonso Herrero ${ }^{3}$, A. Asensio Ramos ${ }^{1}$, J. T. Radomski ${ }^{4}$, \\ C. Packham ${ }^{5}$, R. S. Fisher ${ }^{6}$, and C. M. Telesco
}

${ }^{1}$ Instituto de Astrofísica de Canarias, ${ }^{2}$ University of Kentucky, ${ }^{3}$ Instituto de Estructura de la Materia, ${ }^{4}$ Gemini South Observatory, ${ }^{5}$ University of Florida, and ${ }^{6}$ Gemini North Observatory Email: cra@iac.es

Keywords. galaxies: active, galaxies: nuclei, galaxies: Seyfert, infrared: galaxies

We present subarcsecond resolution mid-infrared (mid-IR) photometry in the range from 8 to $20 \mu \mathrm{m}$ of 18 nearby Seyfert galaxies, reporting high spatial resolution nuclear fluxes for the entire sample (see Table 3 of Ramos Almeida et al. 2009). We construct spectral energy distributions (SEDs) that the AGN dominates, relatively uncontaminated by starlight, adding near-IR measurements from the literature at similar angular resolution. We find that the IR SEDs of intermediate-type Seyferts are flatter and present higher 10 to $18 \mu \mathrm{m}$ ratios than those of Seyfert 2 (Sy2) galaxies.

We fit the SEDs with the clumpy torus models of Nenkova et al. (2008) using the in-housedeveloped BayesClumpy tool (Asensio Ramos \& Ramos Almeida 2009). We find that the clumpy models reproduce the high spatial resolution measurements. Regardless of the Seyfert type, even with high spatial resolution data, near- to mid-IR SED fitting poorly constrains the radial extent of the torus. Because the outer torus contains the coolest material, high angular resolution measurements at wavelengths longer than $15 \mu \mathrm{m}$ are needed to reveal significant variations in the torus size. For the Sy2 galaxies, we find that edge-on geometries are more probable than face-on views, with a number of clouds along equatorial rays of $N_{0}=5-15$ and large values of the torus width $\left(\sigma=\left[50^{\circ}, 75^{\circ}\right]\right)$. For the intermediate-type Seyferts, $N_{0}, \sigma$, and the inclination angle of the torus are lower than those of the Sy2 nuclei. This would imply that the observed differences between type 1 and type 2 AGNs would not be due to orientation effects only, but to different covering factors in their tori. However, due to the limited size of the sample analyzed, these differences are not statistically significant, and a larger sample is needed to confirm it.

The columns of material responsible for the X-ray absorption are larger than those inferred from the model fits for most of the galaxies, which is consistent with $\mathrm{X}$-ray absorbing gas being located within the dust sublimation radius, whereas the mid-IR flux arises from an area farther from the accretion disk. The fits yield both the bolometric luminosity of the intrinsic AGN and the torus-integrated luminosity, from which we derive the reprocessing efficiency of the torus. In the models, the outer radial extent of the torus scales with the AGN luminosity, and we find the tori to be confined to scales less than 5 pc (see Table 9 of Ramos Almeida et al. 2009).

\section{References}

Asensio Ramos, A. \& Ramos Almeida, C. 2009, ApJ, 696, 2075

Nenkova, M., Sirocky, M. M., Nikutta, R., Ivezić, Z., \& Elitzur, M. 2008, ApJ, 685, 160

Ramos Almeida, C., et al. 2009, ApJ, 702, 1127 